Aim of the study: To determine the added value of computed tomography perfusion (CTP) images combined with contrast-enhanced computed tomography (CECT) images in staging of head and neck squamous cell cancer (SCC). Material and methods: Forty-seven consecutive patients with histologically proven squamous cell cancer of the head and neck and qualified for surgical treatment were prospectively evaluated in 2 groups: based on CECT multiplanar reformations (axial, coronal and sagittal), and separately, based on CTP images combined with CECT data. Tumour stage was assessed in each group separately, with special emphasis on T4 stage, and results were compared with histological findings. Five patients underwent endoscopic laser tumour resection, 11 underwent other tumour resection (glossectomy, pharyngectomy) and $31 \mathrm{pa-}$ tients underwent en-bloc resection of the hypopharynx and larynx, allowing detailed pathological evaluation of possible tumour infiltration into surrounding structures. Two experienced head and neck radiologists evaluated the images. Inter-observer agreement was tested with the modified $\kappa$ test. The $\chi^{2}$ test was applied to compare the number of correctly staged tumours for the two methods and readers.

Results: Inter-observer agreement was high $(k=0.88-0.90)$. Significant differences between the two groups were observed; with added CTP assessment more anatomical structures were rated positive for tumour infiltration and diagnostic accuracy of this method was significantly higher when compared to CECT images. Sole evaluation of CECT images in less advanced cases led to overestimation of the disease, since inflammation and slight oedema could not be differentiated from tumour.

Conclusions: Contrast-enhanced computed tomography multi-planar images enhanced with CTP images were proven to improve accuracy in head and neck cancer staging. The added value of CTP may help to avoid overestimation of the malignant process and at the same time may facilitate depiction all infiltrated structures.

Key words: CT perfusion, squamous cell cancer, TNM, computed tomography.

\section{Head and neck squamous cell cancer - the role of computed tomography enhanced with perfusion imaging in tumour staging}

\author{
Agnieszka Trojanowska' ${ }^{1}$ Piotr Trojanowski' ${ }^{2}$ Andrzej Drop ${ }^{3}$, Janusz Klatka
}

${ }^{1}$ Medical University of Lublin

2Department of ENT Surgery, Medical University of Lublin

${ }^{3}$ Department of Radiology, Medical University of Lublin

\section{Introduction}

Multislice computed tomography (MSCT) is presently the workhorse in the evaluation of most head and neck malignancies [1, 2]. For the majority of patients with suspected oropharyngeal and/or laryngeal cancer, MSCT will be the first or the only diagnostic imaging study [2].

Exact tumour staging is necessary for planning the treatment, leading to reduced post-operative morbidity and-most importantly - tumour recurrenceassociated mortality. To date, MSCT with multi-planar reformations (MPR) has been widely used for preoperative staging of head and neck cancer, and its accuracy ranges from $43 \%$ to $89 \%$, depending on the type of scanner [3-6]. Each tumour stage (T1-T4) has its own prognostic importance, but the preoperative prediction of $\mathrm{T} 4$ tumours is of particular significance in determining tumour resectability, optimal extent of surgery and concomitant chemoradiotherapy $[4,7,8]$.

The preoperative accurate discrimination of all infiltrated structures, as well as correct staging, is therefore crucial for surgeons and determines an aggressive or more conservative approach. It is especially true for T4 tumours, where reported MSCT accuracy ranges from as low as 53\% and reaching $90 \%$ in selected cases [4, 8].

During the last decade, functional imaging techniques including computed tomography perfusion (CTP) have emerged as a new tool helpful in the evaluation of malignant tissue, based on the estimation of blood supply kinetics. Computed tomography perfusion has been used extensively in the arena of neurological science and recently it gained interest in oncology [9-11]. This technique is being gradually applied in the diagnosis, staging and prognostic evaluation of tumours, as well as for therapeutic response monitoring [12]. In particular, the latest publications indicate that CTP could provide useful information for differentiation between squamous cell cancer (SCC) and normal tissue [10, 12, 13]. This finding is attributed to the enlarged and hyper-permeable neovascular bed as a consequence of tumoural neoangiogenesis.

Some other imaging techniques also allow for tissue perfusion measurement, but CTP is superior for various reasons, such as widespread availability, affordability, better experience and accuracy of data.

The purpose of this prospective study was to determine the added value of CTP images in routine contrast-enhanced computed tomography (CECT) staging of head and neck cancer, with special regard to T4 cancer staging. 


\section{Material and methods}

\section{Patient selection}

Fifty-six consecutive patients (41 men and 6 women, mean age 49 years) with SCC of the head and neck confirmed on biopsy and considered to be potential candidates for surgery were enrolled in this study over an 18-month period. We excluded from the group patients with $\mathrm{T} 1 \mathrm{glot}-$ tic cancer with no signs of anterior commissure infiltration $(n=6)$, since they did not need CT, and patients with nasopharyngeal cancer $(n=3)$, who were scheduled for magnetic resonance imaging followed by radiotherapy, so finally 47 patients were diagnosed in the Department of Radiology. Written informed consent from all patients and institutional review board approval were obtained. The primary tumour locations were: oral cavity $(n=11)$, oropharynx $(n=7)$, hypopharynx $(n=21)$ and larynx $(n=9)$. Tumour location and stage, based on histopathological reports, are presented in Table 1.

Based on the consensus between imaging findings and clinical evaluation, patients were scheduled for appropriate surgical treatment. Results from CTP examination were not taken into account when planning surgery because of the investigative nature of this study. Of 47 examined patients, all underwent surgery, as follows: endoscopic laser tumour resection $(n=5)$, partial glossectomy $(n=7)$, partial glossectomy with segmental mandibulotomy $(n=1)$, tonsillectomy $(n=1)$, pharyngectomy $(n=2)$ and en bloc resection of hypopharynx and larynx $(n=31)$.

\section{Computed tomography}

Standard contrast-enhanced head and neck study, followed by CTP exam, was performed using a 64-row scanner (VCT, GE Healthcare, Milwaukee, WI, USA) with tube voltage 120 kV, effective tube current 150-170 mAs, collimation 1.25 and rotation time $0.5 \mathrm{~s}$.

First, $80 \mathrm{ml}$ of a non-ionic iodinated contrast agent (iodixanol, 320 mg/ml; Visipaque, GE Healthcare) was injected at a rate of $1 \mathrm{ml} / \mathrm{second}$ and images were acquired with $100 \mathrm{~s}$ delay, from the skull base to the thoracic inlet with $1.25-\mathrm{mm}$ contiguous sections. Two image data sets were reconstructed using a standard soft tissue convolution kernel and sharp bone convolution kernel.

Computed tomography perfusion was performed 5-10 minutes later. For the perfusion study patients received a power injection of $40 \mathrm{ml}$ of the same contrast agent at $4.5 \mathrm{ml} / \mathrm{s}$ and were scanned to acquire 16 contiguous 5 -mm-thick slices (total coverage of $8 \mathrm{~cm}$ ) in CINE mode, for $50 \mathrm{~s}$, at the predetermined levels of interest. The level of interest represented the tumour and in all cases it was possible to include the whole primary lesion. In patients with no defined lesion on the regular enhanced CT scans, perfusion images were obtained through the areas that were clinically suspicious.

\section{Data analysis and reference standard}

Computed tomography $(\mathrm{CT})$ data were independently evaluated by two radiologists with more than 5 years of experience in head and neck radiology, who were blinded to the clinical data and to each other's results.
To reduce the bias introduced by a learning curve or recall of corresponding examinations, data were evaluated in random order and in long time intervals.

A standard workstation was used to read and reformat the images (Advantage Workstation 4.2, GE Healthcare, Milwaukee, WI). The following issues were addressed during evaluation of CECT multi-planar images: space from which the tumour originated, possible infiltration of surrounding structures and staging according to International Union Against Cancer (UICC) guidelines. Signs of malignant infiltration were evaluated as previously described in the literature [14-17].

Computed tomography perfusion images were reconstructed automatically using commercially available software based on the deconvolution method (Perfusion 3, AW 4.2, GE Healthcare, Milwaukee, USA). The following colourcoded maps were obtained: map of blood flow (BF, in $\mathrm{ml} / \mathrm{min} / 100 \mathrm{~g}$ tissue), blood volume map (BV, in $\mathrm{ml} / 100 \mathrm{~g}$ tissue), mean transit time map (MTT, in s) and permeability surface map (PS, in $\mathrm{ml} / \mathrm{min} / 100 \mathrm{~g}$ tissue). For the evaluation of the tumour, a freehand region of interest (ROI) through the primary site (lesion or suspected lesion) was drawn, using the base image; other ROls were placed on surrounding spaces and structures, including muscles, salivary glands, tonsils, mucosa, fat and bone. Mean values of BF, BV, MTT and PS from these structures were compared with values from the contralateral unaffected side. Malignant infiltration was suspected when the values of BF, BV and PS exceeded by $30 \%$ values from contralateral structures, which is in accordance with the results of other authors [18-20].

A detailed description of radiological findings is presented in Table 2.

At histopathological examination, adjacent tissue invasion was regarded as present if tumour cells were identified within that tissue. Invasion was present in a total of 32 structures. In 31 structures the invasion was confirmed on the basis of histopathological examination of the resected specimen, and in the case of carotid space invasion the diagnosis was determined on the basis of the surgical findings because the infiltrated structure could not be resected.

Histological reports were reviewed for the final T stage to validate the results from $\mathrm{CT}$.

\section{Statistical analysis}

A modified Cohen's $\kappa$ test [B] was used to measure the agreement between two readers for both methods of assessment - CECT images and CECT enhanced with CTP. The Wilcoxon signed rank test with continuity correction was applied in order to test the null hypothesis, which postulates the equality of both methods.

Table 1. Tumour localisation and final tumour stage according to histopathological assessment

\begin{tabular}{lccccc} 
Tumour localization & No. of cases & \multicolumn{5}{c}{ Stage } \\
\cline { 3 - 6 } & & T1 & T2 & T3 & T4 \\
\hline oral cavity & 10 & 2 & 5 & 1 & 2 \\
\hline oropharynx & 7 & 1 & 1 & 2 & 4 \\
\hline hypopharynx & 21 & 0 & 1 & 7 & 13 \\
larynx & 9 & 0 & 4 & 3 & 2
\end{tabular}


Table 2. Positive diagnosis of tumour infiltration

\begin{tabular}{|c|c|c|c|c|c|}
\hline Structure & $\begin{array}{c}\text { Reader A } \\
\text { CECT }\end{array}$ & $\begin{array}{c}\text { Reader A } \\
\text { CECT + CTP }\end{array}$ & $\begin{array}{c}\text { Reader B } \\
\text { CECT }\end{array}$ & $\begin{array}{c}\text { Reader B } \\
\text { CECT + CTP }\end{array}$ & Reference standard \\
\hline parotid space & 1 & 1 & 1 & 1 & 1 \\
\hline masticator space & 3 & 4 & 3 & 4 & 4 \\
\hline buccal space & 1 & 1 & 1 & 1 & 1 \\
\hline soft palate & 3 & 3 & 3 & 4 & 5 \\
\hline hard palate & 2 & 2 & 2 & 2 & 2 \\
\hline mandible & 2 & 2 & 2 & 2 & 4 \\
\hline floor of mouth & 9 & 8 & 4 & 8 & 8 \\
\hline sublingual space & 4 & 5 & 4 & 6 & 6 \\
\hline tonsil & 7 & 10 & 8 & 10 & 13 \\
\hline intrinsic tongue muscles & 10 & 14 & 9 & 14 & 14 \\
\hline extrinsic tongue muscles (any) & 3 & 8 & 4 & 7 & 8 \\
\hline tongue base & 3 & 3 & 3 & 4 & 5 \\
\hline submandibular gland & 1 & 1 & 1 & 1 & 1 \\
\hline parapharyngeal space & 1 & 1 & 1 & 1 & 1 \\
\hline carotid space & 4 & 7 & 3 & 7 & 7 \\
\hline pre-epiglottic space & 8 & 8 & 8 & 8 & 8 \\
\hline supraglottic mucosa & 15 & 15 & 13 & 14 & 19 \\
\hline glottic mucosa & 6 & 6 & 6 & 6 & 11 \\
\hline subglottic mucosa & 1 & 1 & 1 & 1 & 3 \\
\hline thyroid cartilage & 2 & 2 & 2 & 2 & 5 \\
\hline cricoid cartilage & 1 & 1 & 2 & 2 & 4 \\
\hline arytenoid cartilage & 4 & 4 & 2 & 2 & 7 \\
\hline true vocal cord & 11 & 12 & 10 & 12 & 13 \\
\hline false vocal cord & 15 & 12 & 12 & 12 & 14 \\
\hline anterior commissure & 3 & 4 & 4 & 4 & 5 \\
\hline pyriform sinus & 17 & 23 & 18 & 22 & 23 \\
\hline posterior hypopharyngeal wall & 5 & 6 & 5 & 5 & 8 \\
\hline prevertebral space & 1 & 1 & 2 & 2 & 1 \\
\hline postcricoid area & 1 & 3 & 2 & 3 & 3 \\
\hline thyroid gland & 2 & 2 & 2 & 2 & 2 \\
\hline laryngeal strap muscles & 3 & 3 & 3 & 3 & 3 \\
\hline oesophagus & 1 & 1 & 1 & 1 & 1 \\
\hline
\end{tabular}

A $\chi^{2}$ test was used to compare the number of tumours classified according to the UICC TNM system correctly for the two methods and each reader. Fisher's exact test was applied to evaluate the relationship between misclassification and the method applied.

\section{Results}

A high level of agreement was found between both readers $(k=0.87$ for CECT and $k=0.89$ for CECT + CTP). Table 2 presents the number of cases in which infiltration of 32 predefined structures was diagnosed by the readers with respect to the method of imaging (CECT vs. CECT + CTP). The most frequent findings were infiltration of tonsil, intrinsic tongue muscle, pyriform sinus, supraglottic mucosa, and true and false vocal cord.

The differences of the scores for both methods showed that evaluation of post-contrast images enhanced with CTP data resulted in a higher score and higher sensitivity in de- 
picting infiltrated structures than evaluation of sole CECT images $(p<0.01)$ (Fig. 1).

In the case of reader 1 , eight patients were down-staged and two patients were up-staged, based on CECT image evaluation, and only 4 patients were down-staged when estimating CECT + CTP images, with no cases of upstaging (Fig. 2A, C). In the case of reader 2 , seven patients were downstaged and 1 patient was up-staged based on evaluation of CECT images, while when estimating CECT + CTP study, only 3 patients were down-staged and 1 patient remained upstaged (Fig. 2B, D).

The structures which were misdiagnosed as non-infiltrated included soft palate, mandible, sublingual space, tonsil, tongue base, laryngeal cartilages and mucosa. Structures which were improperly evaluated as infiltrated included floor of the mouth muscles and false vocal cord.

Detailed evaluation of the possible infiltration of structures specific for stage T4, including carotid space, mandible, thyroid/cricoid cartilages and extrinsic tongue muscles, was performed separately. Carotid space infiltration was much better detectable with the aid of CTP $(p<0.01)$, allowing correct diagnosis in $100 \%$ of cases (Fig. 3A). On the other hand, neither CECT images nor the additional information from CTP study helped in depicting infiltration of mandible and laryngeal cartilages (Fig. 3B, D) - in 2 cases out of 4, the involvement of the mandible was not diagnosed, and in 5 cases out of 9 , malignant changes in thyroid/cricoid cartilages were not seen
Table 3. Fisher's exact test for number of misclassified stages between CECT and CECT + CTP methods

\begin{tabular}{lcccccccc} 
Method & \multicolumn{4}{c}{ Reader A } & \multicolumn{3}{c}{ Reader B } \\
\cline { 2 - 9 } & T1 & T2 & T3 & T4 & T1 & T2 & T3 & T4 \\
CECT & 0 & 2 & 3 & 5 & 0 & 1 & 4 & 4 \\
CECT + CTP & 0 & 0 & 2 & 2 & 0 & 1 & 1 & 2
\end{tabular}

in imaging studies. In the case of extrinsic tongue muscle infiltration, the addition of CTP resulted in correct diagnosis in all cases for reader $A$ and only one misdiagnosed case for reader $\mathrm{B}$ (Fig. 3C).

Agreement between CT-based T stage and the final T stage was high, with the exception of stage $\mathrm{T} 4$, since in this group, based on CECT images, more than $50 \%$ of important infiltrated structures were not depicted. The added role of CTP had the greatest impact on staging precisely in this group, and this examination enabled $100 \%$ of correct diagnoses in cases of soft tissue invasion (carotid space and tongue muscles).

\section{Discussion}

In many head and neck squamous cell cancers, the visible mucosal lesion, evaluated clinically, represents the tip of an iceberg since submucosal spread is frequent, skip behaviour occurs and deep soft tissue involvement has been proven in many advanced cases [1, 4, 8].
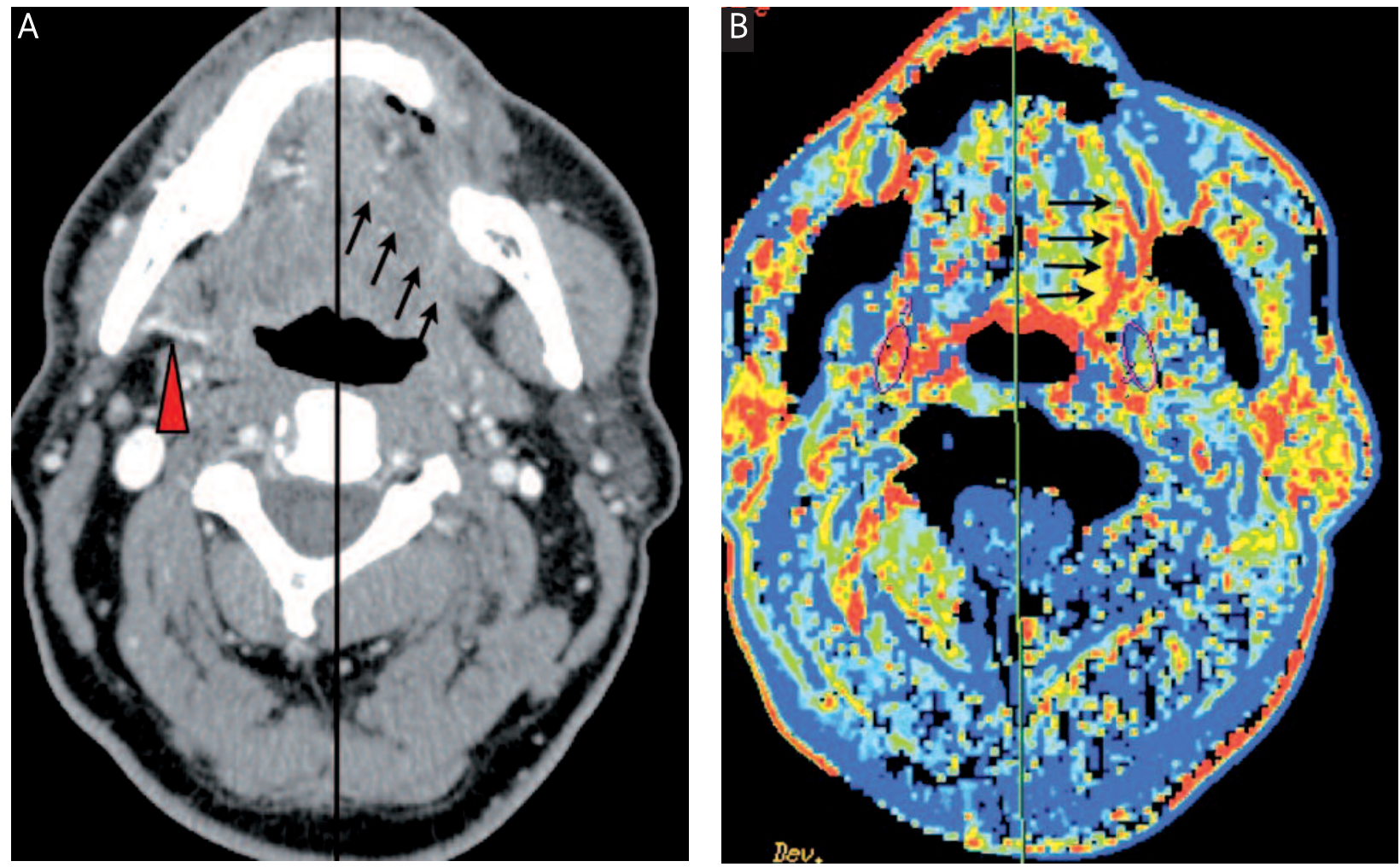

Fig. 1. A 48-year-old man presenting with squamous cell cancer of the left side of the tongue. A) Axial CECT image shows no visible pathological contrast enhancement and no signs of floor of the mouth involvement on the left side (arrows), which is symmetrical, compared to the right side. B) Axial CTP map of blood volume demonstrates area with high blood volume values (arrows), which is consistent with malignant infiltration of the floor of the mouth. This finding was confirmed during surgery. Note the asymmetric course of the facial artery (red arrow, A), which may be the source of false-positive results on CTP maps - part of the pharyngeal mucosal space on the right side on the blood volume map (B) has higher blood volume values compared to the contralateral side (two purple ROIs on both sides) - this is due to the partial volume effect. 
A

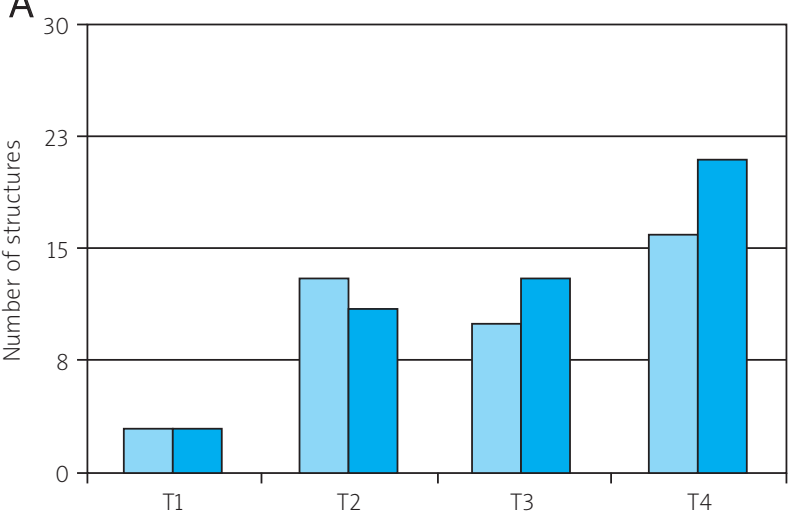

C

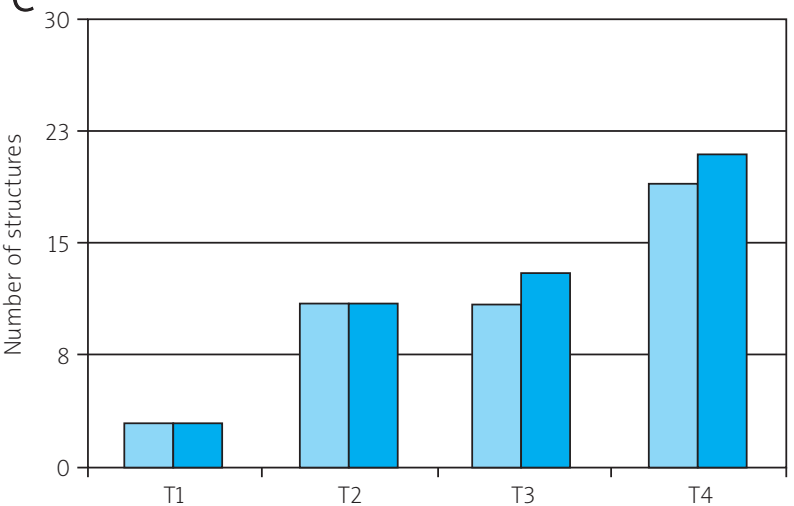

B

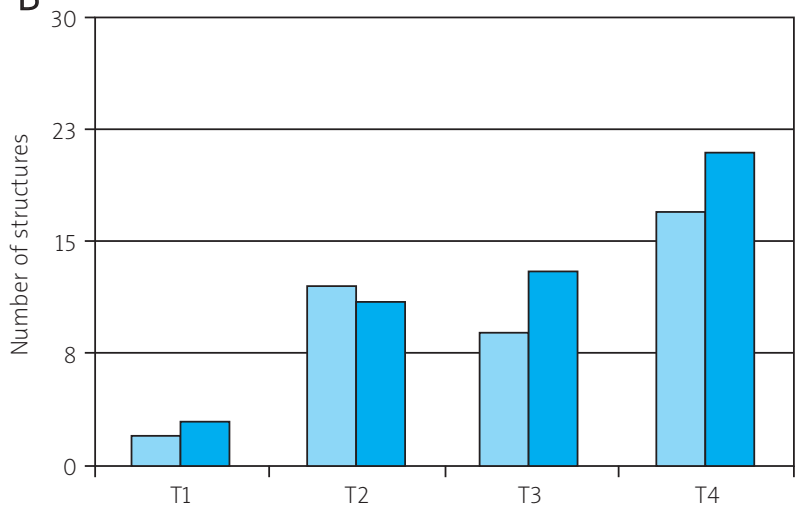

D

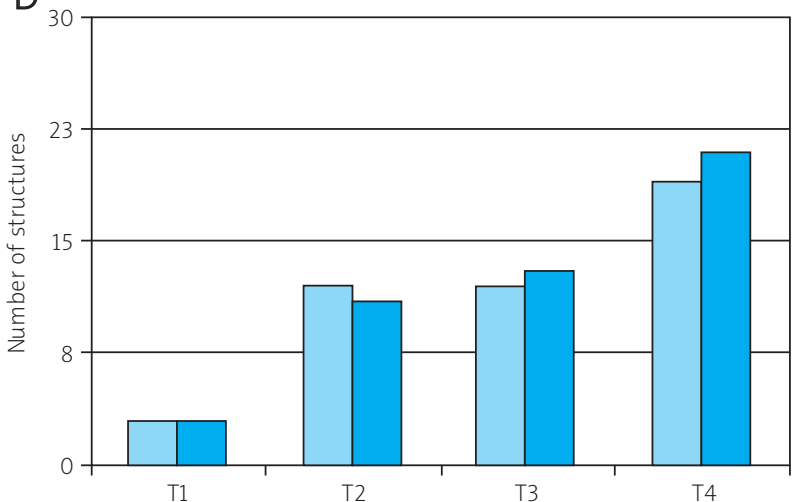

$A-D$, graphs show results for reader $A$ when evaluating $C E C T$ images $(A)$, reader $B$ using $C E C T$ images $(B)$, reader $A$ using CECT + CTP images (C) and reader $B$ using CECT + CTP images (D)

Fig. 2. Structures which were classified as infiltrated according to the reader (light blue), and structures which were proven to be infiltrated according to the results of histopathological examination (dark blue)

Presently there is no doubt that imaging studies should be performed in order to evaluate the extent of tumour and to stage it correctly $[2,8]$.

Since MSCT has become the first-line examination for most pathologies, tumour staging will be proposed and appropriate treatment will be considered based on computed tomography findings. Also, critical issues such as carotid space encasement, prevertebral fascia involvement, bone infiltration and extrinsic tongue muscle infiltration will have to be answered accurately, since head and neck surgeons more and more rely on imaging studies, in order to determine resectability of the tumour. These crucial questions have to be addressed to model an appropriate therapy concept.

However, the low tissue resolution of computed tomography is a well-known drawback of this method. Based on CECT studies, sometimes it may be difficult to differentiate between tumour and inflammation or oedema, which usually leads to upstaging of the malignant process $[6,8,16]$. For better evaluation of the extent of malignant tissue, a new tool of functional imaging has been introduced - CT perfusion. Starting from brain ischaemia and brain tumour evaluation [3], it quickly became an effective, reliable and accurate method for the assessment of neo-angiogenesis, which is typical for malignancy [9-11].
Based on the visualisation and mathematical evaluation of tissue perfusion parameters, CTP has already proven its ability to differentiate tumour from normal tissue [18, 19].

At present, CTP examination can be completed in a couple of minutes on every MSCT scanner, and perfusion maps are generated automatically, so the addition of CTP into routine CT examination of head and neck is neither time-consuming nor demanding.

The aim of our study was to determine the added value of CTP images combined with CECT images in staging of head and neck squamous cell cancer, with special emphasis on evaluating T4 tumours.

Both of our readers showed a high level of agreement for each evaluation method.

With regard to previous studies [10, 11, 17], we hypothesised that in order to achieve high accuracy in defining the extent of malignancy, the study protocol would benefit from an additional functional imaging method - CTP. In agreement with this hypothesis, more anatomical structures were correctly rated to be invaded by tumour with CECT + CTP compared with evaluation of sole CECT images.

On the other hand, when evaluating $T 1$ tumours, basic contrast-enhanced images were sufficient to stage the tumour correctly in all cases. These findings are in agreement with those of Lell et al. [20], who proved that, especially in less 
A

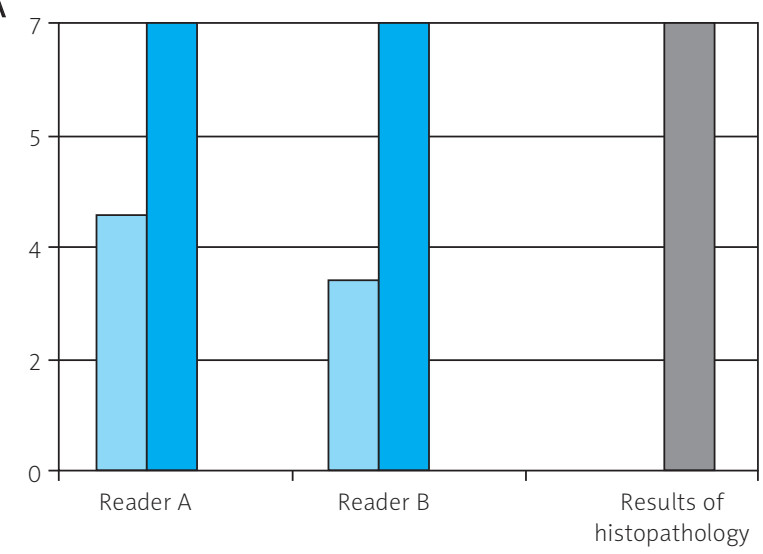

C

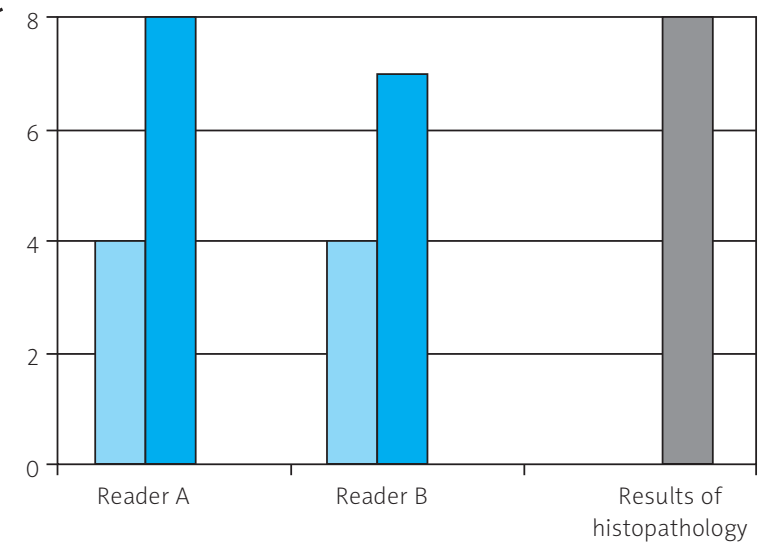

B

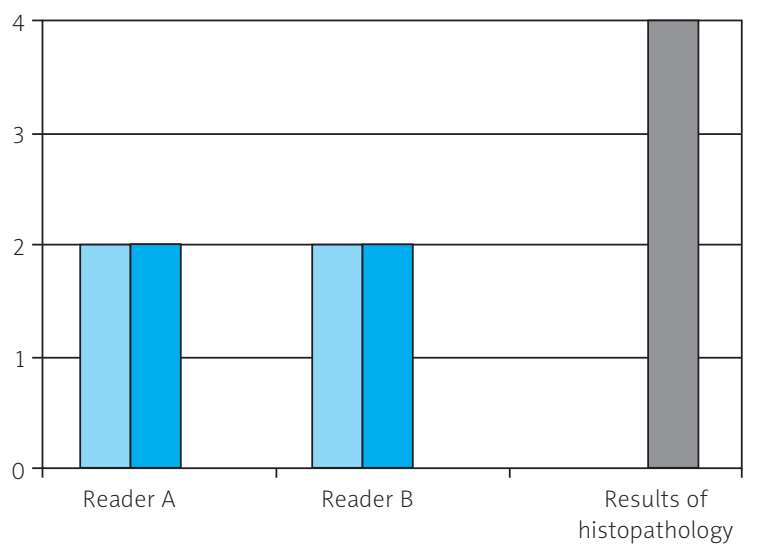

D

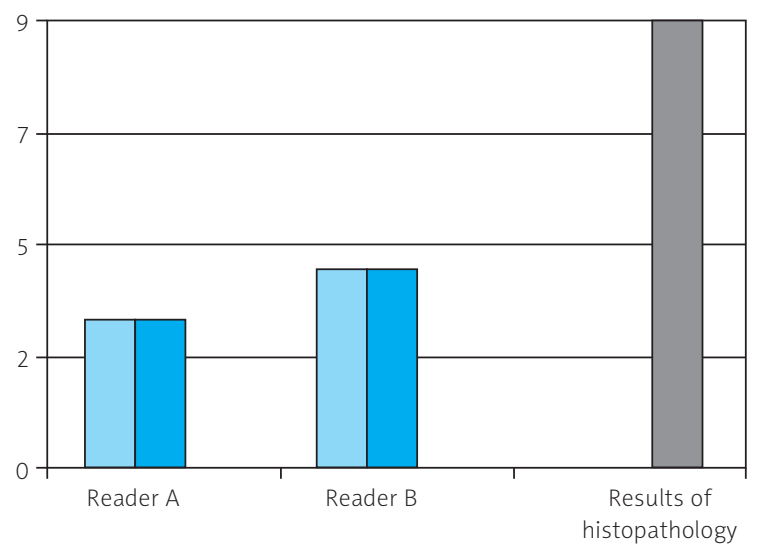

$\square$ CECT $\quad \square$ CECT + CTP $\square$ histopathology

Fig. 3. Evaluation of different features specific for T4 stage (A-D) - comparison of CECT, CECT + CTP and histopathology for reader A and reader B. Cases of infiltration depicted based on CECT are presented in light blue, cases depicted based on CECT + CTP are presented in dark blue, and cases confirmed on histopathology are presented in grey

A - Cases diagnosed with carotid space infiltration

$B$ - Cases with mandibular infiltration

C - Cases of extrinsic tongue muscle infiltration

D - Cases of thyroid and/or cricoid cartilage infiltration

advanced stages, standard contrast-enhanced axial images are as accurate as other sophisticated CT reformations.

Interesting was the fact that in some less advanced tumours, mainly oral cavity cancers, assessment of CECT images led to upstaging of the process, since some structures, mainly floor of the mouth muscles, were rated as infiltrated. This observation may be explained by the theory that inflammation and slight oedema, which accompanies the tumoural growth and affects surrounding structures, is indistinguishable from malignant tissue itself in standard CT examination. It requires functional imaging techniques to differentiate between malignant and non-malignant processes, based on blood flow and blood volume values. This observation is in line with those reported by Becker et al. [14], who pointed out the tendency of CT to up-stage malignant tumours in a large number of cases.

The results of our study show that in this doubtful group, analysis of CTP images may help to clarify the true nature of lesions visible on CECT images.

In our study we have proven CTP to be a sensitive and accurate tool in defining infiltration of muscles, with special at- tention paid to extrinsic tongue muscles. We observed that in infiltrated muscles, BF, BV and PS values were almost twice as high as in non-infiltrated contralateral structures. This observation is in agreement with previous studies of Bisdas et al. $[13,17]$, who observed significantly lower $(p<0.001)$ perfusion values for unchanged tongue, paraspinous and sternocleidomastoid muscles, in comparison to the pathological contralateral site. This finding also supports the thesis that perfusion imaging may be a powerful tool for imaging activity of the tumour and its infiltrative pattern, before the tumour proceeds in a gross anatomical distortion.

Of course, perfusion is not a universal tool - one should be very careful in evaluation of mucosal or very shallow submucosal spread of tumour based on CTP values, since the upper aerodigestive tract mucosa is very well perfused, CTP values are very high, and such evaluation is best performed on endoscopy [8].

Also, similar inability to differentiate between healthy and infiltrated tissue based on CTP images has been shown in this study for bones. In bone, blood flow and blood volume values are very low (due to the histological type of tissue), 
and even visible disturbances in terms of osteosclerosis and osteolysis do not produce a noticeable change in any of the perfusion parameters. This was well illustrated during a separate analysis of mandibular and cartilaginous infiltration in the T4 group, and is also in line with previous reports $[3,14]$ indicating that accurate evaluation of bone in terms of possible infiltration requires both $\mathrm{CT}$ and MR imaging.

Our observations also showed that when infiltration encased lymphoid tissue (tonsillar and tongue base cancer), it was difficult to define the exact borders of the tumour, because of physiological contrast enhancement of tonsils. In these particular cases, BF, BV and PS values allowed for better delineation of the pathological process, since blood flow almost doubles in the case of malignant tonsillar infiltration.

One of the major advantages of CTP, according to our results, was the ability to visualise pathological tissue in the carotid space, since every case was confirmed intra-operatively by a surgeon, or histopathologically. Infiltration of the carotid space is a very powerful negative predictive factor and makes total resectability of the tumour very doubtful. The carotid space is bordered by all three layers of the deep cervical fascia; its invasion and presence of even a minimal amount of malignant tissue within implies extremely high probability of recurrence [4].

In conclusion, it can be stated that CECT examination enhanced with CTP images has the potential to improve accuracy in head and neck cancer staging. The added value of CTP may help to avoid overestimation of the malignant process and at the same time may facilitate depiction of the infiltration of some crucial structures, such as the carotid space, extrinsic tongue muscles, and floor of the mouth muscles. However, possible bone and cartilage invasion is not evident on CTP and this limitation should be considered.

\section{Acknowledgements}

This study was entirely financed by State Committee for Scientific Research grant number N N402 352538.

\section{References}

1. Stambuk HE, Karimi S, Lee N, Patel SG. Oral cavity and oropharynx tumors. Radiol Clin North Am 2007; 45: 1-20.

2. Shah GV, Wesolowski JR, Ansari SA, Mukherji SK. New directions in head and neck imaging. J Surg Oncol 2008; 97: 644-48.

3. Emonts P, Bourgeois P, Lemort M, Flamen P. Functional imaging of head and neck cancers. Curr Opin Oncol 2009; 21: 212-7.

4. Conley BA. Treatment of advanced head and neck cancer: what lessons have we learned? J Clin Oncol 2006; 24: 1023-5.

5. Marur S, Forastiere AA. Head and neck cancer: changing epidemiology, diagnosis, and treatment. Mayo Clin Proc 2008; 83: 489-501.

6. Dammann F, Horger M, Mueller-Berg M, Schlemmer H, Claussen CD, Hoffman J, Eschmann S, Bares R. Rational diagnosis of squamous cell carcinoma of the head and neck region: comparative evaluation of CT, MRI, and 18FDG PET. AJR Am J Roentgenol 2005; 184: 1326-31.

7. Petralia G, Preda L, Giugliano G, et al. Perfusion computed tomography for monitoring induction chemotherapy in patients with squamous cell carcinoma of the upper aerodigestive tract: correlation between changes in tumor perfusion and tumor volume. I Comput Assist Tomogr 2009; 33: 552-9.

8. Mukherii SK, Pillsbury HR, Castillo M. Imaging squamous cell carcinomas of the upper aerodigestive tract: what clinicians need to know. Radiology 1997; 205: 629-46.
9. Miles KA. Tumour angiogenesis and its relation to contrast enhancement on computed tomography. Eur J Radiol 1999; 30: 198-205.

10. Rumboldt Z, Al-Okaili R, Deveikis JP. Perfusion CT for head and neck tumours: pilot study. AJNR Am J Neuroradiol 2005; 26: 1178-85.

11. Hermans R, Lambin P, Van der Goten A, Van den Bogaert W, Verbist B, Weltens C, Delaere PR. Tumoural perfusion as measured by dynamic computed tomography in head and neck carcinoma. Radiother Oncol 1999; 53: 105-11.

12. Hermans R, Meijerink M, Van den Bogaert W, Rijnders A, Weltens C, Lambin P. Tumour perfusion rate determined noninvasively by dynamic computed tomography predicts outcome in head-and-neck cancer after radiotherapy. Int J Radiat Oncol Biol Phys 2003; 57: 1351-6.

13. Bisdas S, Baghi M, Wagenblast J, Knecht R, Thng CH, Koh TS, Vogl TJ. Differentiation of benign and malignant parotid tumors using deconvolution-based perfusion CT imaging: feasibility of the method and initial results. Eur J Radiol 2007; 64: 258-65.

14. Becker M. Neoplastic invasion of laryngeal cartilage: radiologic diagnosis and therapeutic implications. Eur J Radiol 2000; 33: 216-29.

15. Harnsberger HR, Wiggins RH, Hudgins PA, et al. Diagnostic imaging: head and neck. AMIRSYS, Salt Lake City UT 2004.

16. Lenz M, Greess H, Baum U, Dobritz M, Kersting-Sommerhoff B. Oropharynx, oral cavity, floor of the mouth: CT and MRI. Eur J Radiol 2000; 33: 203-15.

17. Bisdas S, Baghi M, Smolarz A, et al. Quantitative measurements of perfusion and permeability of oropharyngeal and oral cavity cancer, recurrent disease, and associated lymph nodes using first-pass contrast-enhanced computed tomography studies. Invest Radiol 2007; 42: 172-9.

18. Gandhi D, Hoeffner EG, Carlos RC, Case I, Mukherji SK. Computed tomography perfusion of squamous cell carcinoma of the upper aerodigestive tract: initial results. J Comput Assist Tomogr 2003; 27: 687-93.

19. Faggioni L, Neri E, Cerri F, Picano E, Seccia V, Muscatello L, Franceschini SS, Bartolozzi C. 64-row MDCT perfusion of head and neck squamous cell carcinoma: technical feasibility and quantitative analysis of perfusion parameters. Eur Radiol 2011; 21: 113-21.

20. Lell MM, Gmelin C, Panknin C, Eckel KT, Schmid M, Bautz WA, Greess $\mathrm{H}$. Thin slice MDCT of the neck: impact on cancer staging. AJR Am J Roentgenol 2008; 190: 785-9.

\section{Address for correspondence}

\section{Agnieszka Trojanowska}

Medical University of Lublin

Jaczewskiego 8

20-090 Lublin, Poland

e-mail: agnieszka30@yahoo.com 